\title{
On the use of simple classifiers for the initialisation of one-hidden-layer neural nets
}

\author{
Jan C. Bioch, Robert Carsouw, Rob Potharst \\ Department of Computer Science \\ Erasmus University Rotterdam \\ P.O. Box 1738,3000 DR Rotterdam \\ The Netherlands \\ \{bioch, robp\}@cs.few.eur.nl \\ Technical Report eur-cs-95-08
}

\begin{abstract}
In this report we discuss the use of two simple classifiers to initialise the input-tohidden layer of a one-hidden-layer neural network. These classifiers divide the input space in convex regions that can be represented by membership functions. These functions are then used to determine the weights of the first layer of a feedforward network.
\end{abstract}

Keywords and phrases: mapping decision trees onto neural networks, simple perceptrons, LVQ-networks, initialisation of feedforward networks.

\section{Introduction}

In this report we discuss how two well-known classifiers can be used to initialise the first layer of a neural network. These classifiers divide the input space in convex regions. These regions are represented by so-called membership functions generated during training. Subsequently, the coefficients of these membership functions are used to initialise (the weights of) a neural network. This is called a mapping of the classifiers onto the neural net. The neural net is then further trained to improve the performance of the classifier. According to Park [Par94] such a mapping results in a faster convergence of the neural net and in avoiding local minima in network training. In general these mappings are also interesting because they determine an appropriate architecture of the neural net.

In this report we mainly discuss a mapping of a linear tree classifier (LTC) onto a feedforward neural net classifier (NNC) with one hidden layer. The LTC used here is a hierarchical classifier that employs linear functions at each node in the tree. For the construction of decision trees we refer to [PaS90, FaI92, Qui93]. It is known that both the LTC and NNC classifier share the property of universal approximation, i.e both classsifiers are able to solve arbitrary classification problems by forming complex decision boundaries in the feature space. Several authors [Set90, IKP94, Par94] discuss the mapping of an LTC onto a feedforward net with one or two hidden layers, see also [Car95]. A discusion of a mapping onto a net 
with two hidden layers can be found in Sethi [Set90] and Ivanova\&Kubat [IKP94]. A mapping onto a net with one hidden layer is discussed in Park [Par94]. However, the question of how to map an LTC directly on a neural net with one hidden layer is still open. Park [Par94] has suggested an interesting approach of such a mapping based on representing the convex regions induced by an LTC by linear membership functions. However, in Park [Par94] no explicit expression for the coefficients of the membership functions is given. These coefficients depend on a parameter $\rho$ that has to be supplied by the user. In section 3 we show that in general it is not possible to find linear membership functions that represent the convex regions induced by an LTC. It is however possible to find subregions that can be represented by linear membership functions. We derive explicit expressions for the aforementioned parameter $\rho$, in section 4 . This makes it possible to control the approximation of the convex regions by membership functions and therefore of the initialisation of the neural net. In section 5 we discuss the use of LVQ-networks to initialise a feedforward network. It appears that unlike an LTC, an LVQ-network divides the input space in convex regions that can be represented by linear membership functions.

\section{Mapping decision trees to neural nets}

Suppose we are given a multivariate decision tree $(\mathcal{N}, \mathcal{L}, \mathcal{D})$. In this notation, $\mathcal{N}$ is the set of nodes of the tree, $\mathcal{L}$ is the set of leaves of the tree and $\mathcal{D}$ is the set of linear ${ }^{1}$ functions $d_{k}: \mathbb{R}^{n} \rightarrow \mathbb{R}, k \in \mathcal{N}$. In any node $k$ of the tree, the linear function $d_{k}$ is used to decide which branch to take. Specifically, we go left if $d_{k}(x)>0$, right if $d_{k}(x) \leq 0$, see figure 1 .

A decision tree induces a partitioning of $\mathbb{R}^{n}$ : each leaf $\ell$ corresponds with a convex region $R_{\ell}$, which consists of all points $x \in \mathbb{R}^{n}$, that get assigned to leaf $\ell$ by the decision tree. For example, region $R_{5}$ consists of all $x \in \mathbb{R}^{n}$ with $d_{1}(x) \leq 0$ and $d_{3}(x) \leq 0$.
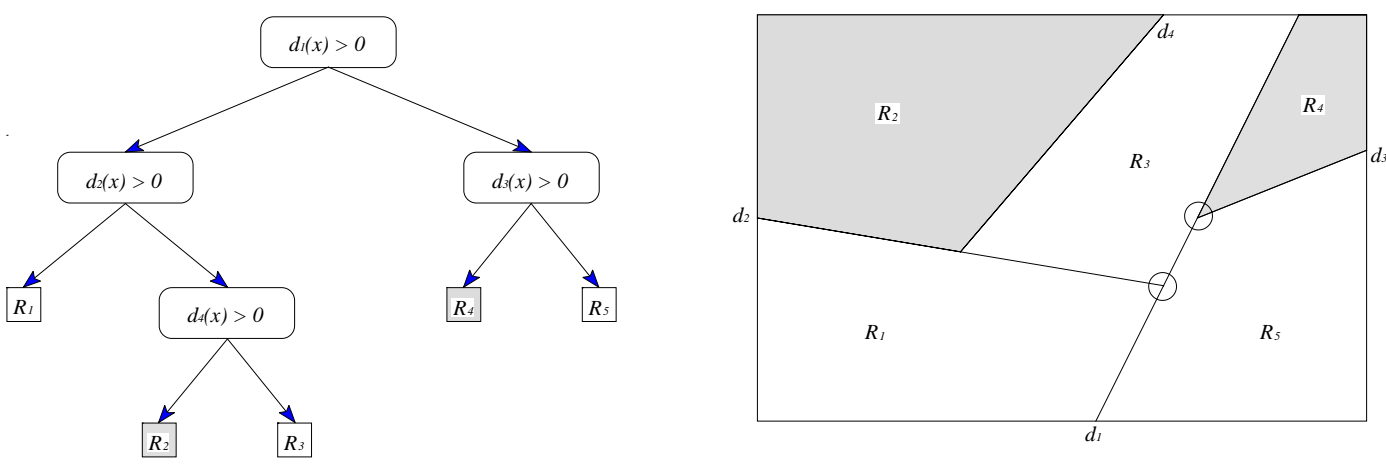

Figure 1: The convex regions induced by a classification tree

In [Set90] Sethi shows a straightforward mapping of an LTC onto an NNC with two hidden layers, see figure 2. In this approach the coefficients of the linear decision functions are used to define the weights in the input to partition layer. Each node in the partition layer

\footnotetext{
${ }^{1} \mathrm{~A}$ linear function is understood to be a function with the following form $: d_{k}(x)=\sum_{j=1}^{n} w_{k j} x_{j}+w_{k 0}$, for $x=\left(x_{1}, \ldots, x_{n}\right) \in \mathbb{R}^{n}, k \in \mathcal{N}$.
} 
corresponds to a decision node in the tree. The nodes in the AND-layer correspond with the decision regions induced by the LTC. Each node in the AND-layer represents a path from the root to a leaf. Subsequently, this decision regions are joined in the OR-layer, to represent the classes. An exact mapping employs threshold units in the neural net. However, as shown in [Set90] continuous transfer functions such as the sigmoid function are more appropriate. Therefore the mapping is only used as an initialisation of a two-hidden-layer neural net. An important feature of Sethi's mapping is that it solves the credit assignment problem for the hidden layers. Therefore it is possible to train the two layers separately. For another interesting approach of an LTC to NNC mapping we refer to [IKP94]. Although a direct mapping onto a one-hidden-layer is not yet known, Park [Par94] describes an approach to initialise the weights in the input to hidden layer. In this approach, discussed in sections 3 and 4 the coefficients of the linear membership functions are obtained by combining the decision functions employed by the tree to define the aforementioned weights, see figure 3 .

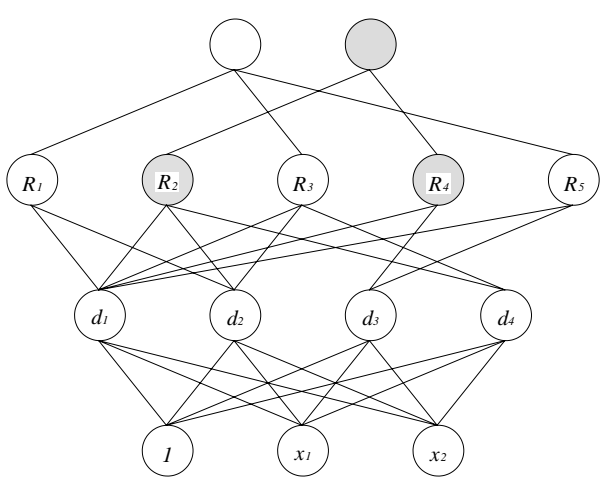

Figure 2: Sethi's mapping

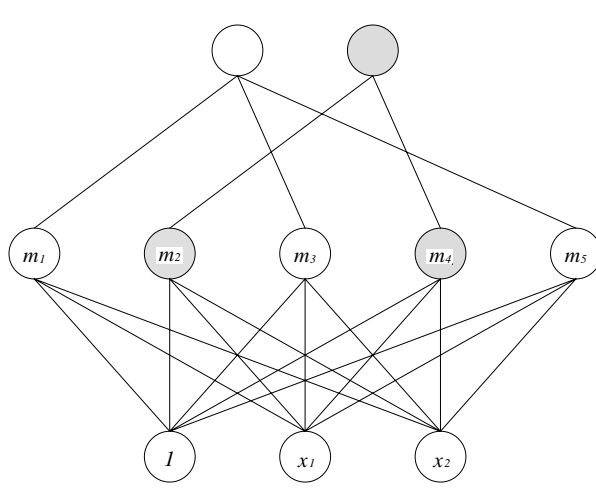

Figure 3: Park's mapping

\section{Non-existence of linear membership functions for LTCs}

In this section we discuss the idea of linear membership functions to represent the convex regions induced by an LTC, and we show that these functions are in general not possible.

In [Par94] the following 'theorem' is given without proof, though supplied with heuristic reasoning for its plausibility, see also equation 5 in the next section:

Conjecture (Park[Par94]) For every decision tree $(\mathcal{N}, \mathcal{L}, \mathcal{D})$ there exists a set of linear membership functions $M=\left\{m_{\ell}, \ell \in \mathcal{L}\right\}$, such that for any $\ell, \ell^{\prime} \in \mathcal{L}$, with $\ell \neq \ell^{\prime}$ :

$$
m_{\ell}(x)>m_{\ell^{\prime}}(x), \forall x \in R_{\ell} .
$$

We first will refute this conjecture by giving a (smallest) counterexample. Then we show that in general linear membership functions cannot exist, and finally we discuss the possibility of polynomial membership functions.

Set $n=2$; we then have points $(x, y) \in \mathbb{R}^{2}$ as our decision vectors. Now, consider the following decision tree $(\mathcal{N}, \mathcal{L}, \mathcal{D}): \mathcal{N}=\{1,2\}, \mathcal{L}=\{1,2,3\}$ and $d_{1}(x, y)=x, d_{2}(x, y)=y$. 
This decision tree and its corresponding partitioning of the sample space are shown in figure 4:

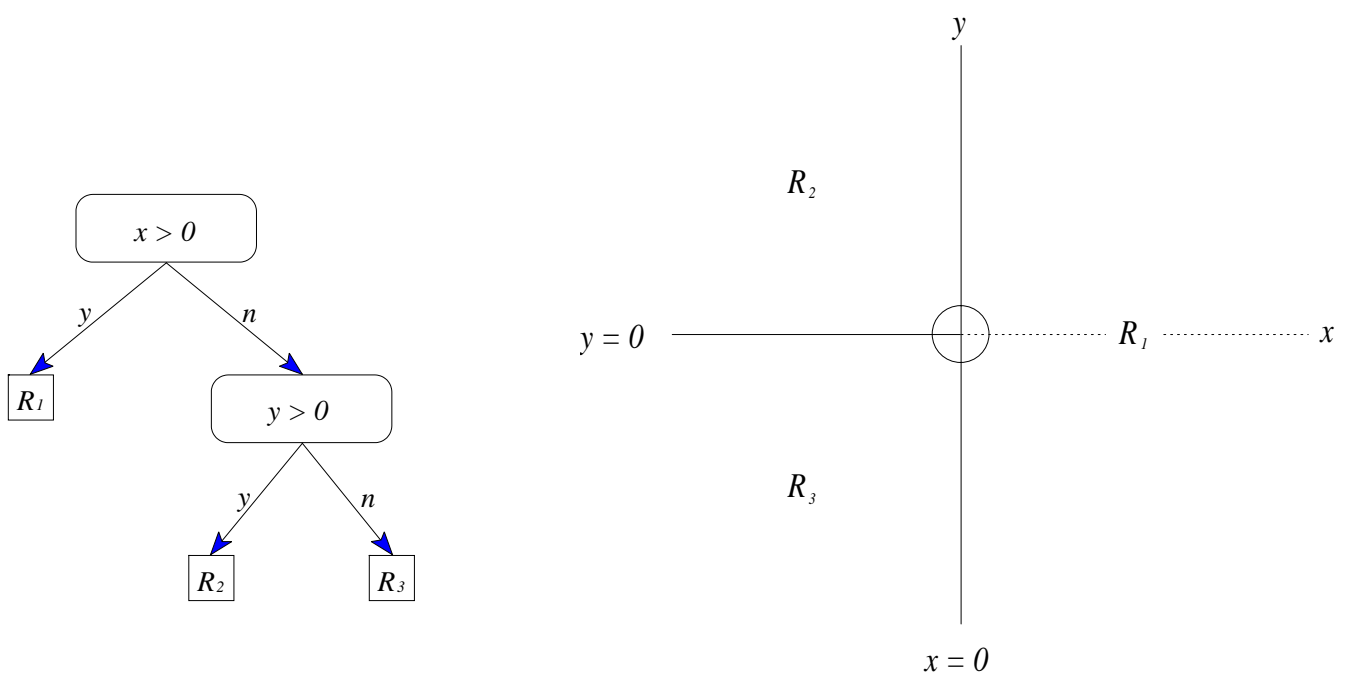

Figure 4: The simplest counterexample to Park's conjecture

Suppose now, that linear functions $m_{1}, m_{2}$ and $m_{3}$ exist, for which the inequality (1) is true. We then have

$$
\begin{aligned}
& m_{1}=p_{1} x+q_{1} y+r_{1} \\
& m_{2}=p_{2} x+q_{2} y+r_{2} \\
& m_{3}=p_{3} x+q_{3} y+r_{3}
\end{aligned}
$$

with

$$
\begin{aligned}
& m_{1}>m_{2} \text { and } m_{1}>m_{3} \text { for all }(x, y) \in R_{1} \\
& m_{2}>m_{1} \text { and } m_{2}>m_{3} \text { for all }(x, y) \in R_{2} \\
& m_{3}>m_{1} \text { and } m_{3}>m_{2} \text { for all }(x, y) \in R_{3} .
\end{aligned}
$$

For the linear function $u(x, y)=m_{1}-m_{2}=\left(p_{1}-p_{2}\right) x+\left(q_{1}-q_{2}\right) y+\left(r_{1}-r_{2}\right)$ we then see that it is positive on $R_{1}$ and negative on $R_{2}$. Consequently, $u=0$ on the borderline between $R_{1}$ and $R_{2}$, which is the vertical axis. It is then easy to see that

$$
p_{1}>p_{2}, q_{1}=q_{2} \text { and } r_{1}=r_{2}
$$

must hold.

If next we consider the linear function $v(x, y)=m_{1}-m_{3}=\left(p_{1}-p_{3}\right) x+\left(q_{1}-q_{3}\right) y+\left(r_{1}-\right.$ $\left.r_{3}\right)$, it will be seen that $v$ is positive on $R_{1}$ and negative on $R_{3}$. Analogously, we then find that

$$
p_{1}>p_{3}, q_{1}=q_{3} \text { and } r_{1}=r_{3} .
$$

By combining (2) and (3) we find that $u-v=m_{3}-m_{2}=\left(p_{3}-p_{2}\right) x$ and, consequently,

$$
\left(p_{3}-p_{2}\right) x>0 \text { on } R_{3} \text {, and }<0 \text { on } R_{2} \text {. }
$$


For points $(x, y) \in R_{2} \cup R_{3}$, we have $x<0$. Thus, it follows from (4) that both $p_{3}<p_{2}$ and $p_{3}>p_{2}$ must be true, which is a contradiction. We must conclude that the hypothesised functions $m_{1}, m_{2}$ and $m_{3}$ cannot exist. Thus, the conjecture is refuted.

Remark. If the decision functions $d_{1}, d_{2}, \ldots$ are chosen in such a way that the hyperplanes $d_{i}(x)=0$ are parallel, then in general it will be possible to construct the above mentioned $m$-functions. For example, in $\mathbb{R}^{2}$ it can be done as follows:

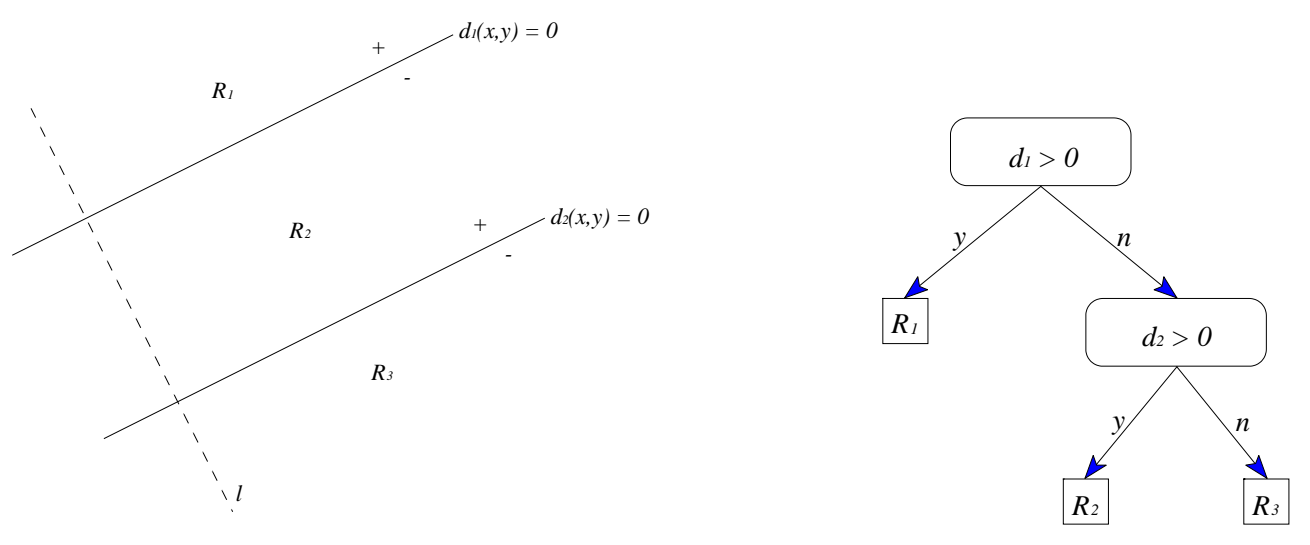

Figure 5: A decision tree with parallel decision boundaries

The graphs of the functions $m_{1}, m_{2}$ and $m_{3}$, intersected with a plane through $\ell$ ( $=$ a perpendicular to $d_{1}$ and $d_{2}$ ) then look as shown in figure 6 :

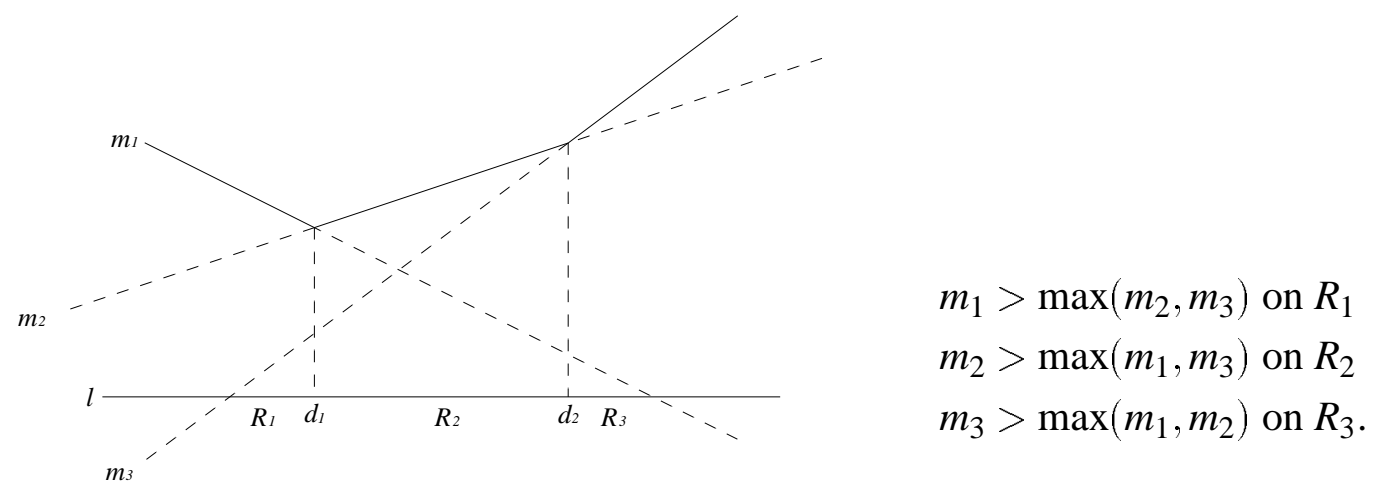

Figure 6: A solution in the case of parallel decision boundaries

In the next theorem we show that linear membership functions that represent the convex regions induced by an LTC cannot exist.

Theorem 1 Let $(\mathcal{N}, \mathcal{L}, \mathcal{D})$ be a decision tree, with at least two non-parallel decision boundaries. Then the convex regions induced by this tree cannot be represented by a set of linear membership functions.

Proof Let $d_{1}$ and $d_{2}$ be two $n$-dimensional linear decision functions such that the hyperplanes $d_{1}=0$ and $d_{2}=0$ are non-parallel. Then for our discussion it is no restriction to consider only the convex regions $R_{1}, R_{2}$ and $R_{3}$ induced by these hyperplanes, see figure 7 (left side). The corresponding membership functions of these regions are denoted by $m_{1}, m_{2}$ 
and $m_{3}$, and the differences $m_{1}-m_{2}, m_{1}-m_{3}$ and $m_{2}-m_{3}$ are respectively denoted by $u$, $v$ and $w$. By definition we have: $u>0$ on $R_{1}$ and $u<0$ on $R_{2}$. Since linear functions are continuous, it is easy to see that the inequalities imply that the linear function $u$ is zero on the hyperplane $d_{1}=0$. Similarly it follows that the function $v$ is zero on $d_{1}=0$ and that $w$ is zero on $d_{2}=0$. Since $w=u-v, w$ is also zero on $d_{1}=0$. Using the fact that $w$ is a linear function which is zero on two non-parallel hyperplanes we conclude that $w$ is identical to the zero-function. This contradicts the fact that $w>0$ on $R_{2}$

Having shown that, in general, linear membership functions cannot represent the convex regions induced by an LTC, the question now arises whether membership functions of LTCs can be represented by multivariate polynomials. The following theorem shows that these polynomials cannot be quadratic.

Theorem 2 Let $(\mathcal{N}, \mathcal{L}, \mathcal{D})$ be a decision tree, with at least two non-parallel decision boundaries. Then the convex regions induced by this tree cannot be represented by a set of quadratic polynomials.

Proof Let $R_{1}, R_{2}$ and $R_{3}$ be the regions induced by a decision tree, see figure 7 (left side).
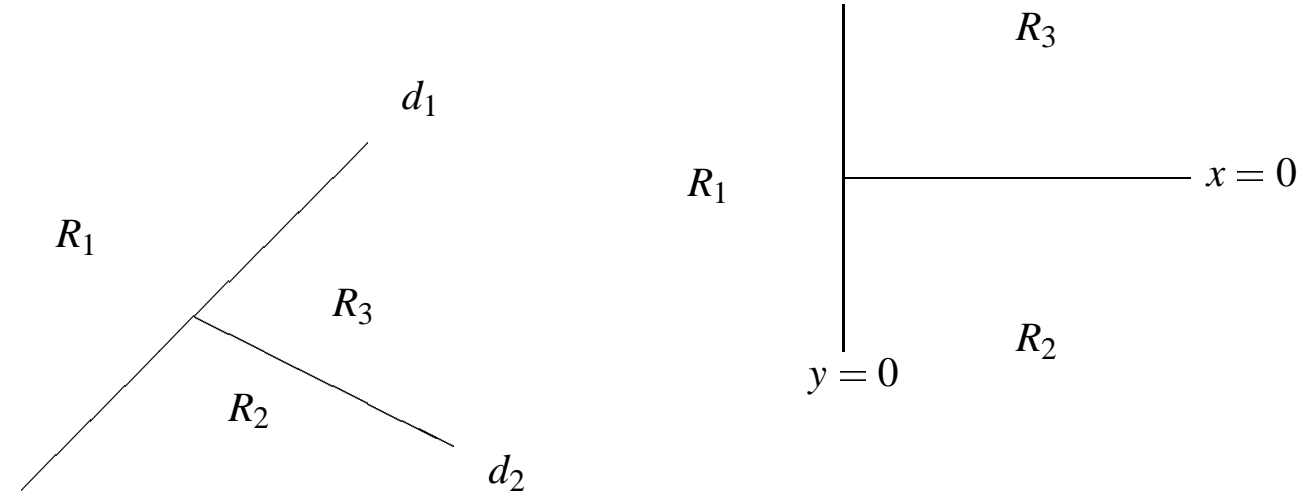

Figure 7: Decision regions in space

Note that such regions will always be induced by a subtree of a decision tree, unless all decision boundaries are parallel. We assume that the regions $R_{1}$ and $R_{2} \cup R_{3}$ are separated by the hyperplane $d_{1}(x)=0, x \in \mathbb{R}^{n}$ such that $d_{1}(x)>0$ on $R_{1}$ and $d_{1}(x)<0$ on $R_{2} \cup R_{3}$. Similarly, $R_{2}$ and $R_{3}$ are separated by $d_{2}(x)=0$, such that $d_{2}(x)>0$ on $R_{2}$ and $d_{2}(x)<0$ on $R_{3}$. Let $m_{1}, m_{2}$ and $m_{3}$ respectively denote the membership functions of $R_{1}, R_{2}$ and $R_{3}$. By definition $m_{1}=0$ on the hyperplane $d_{1}(x)=0$. Similarly, $m_{2}=0$ on $d_{2}(x)=0$. (Note, that we actually know only that $m_{2}$ is zero on half of the hyperplane $d_{2}(x)=0$. However, using a simple result from algebraic geometry it follows that $m_{2}$ must be zero on the whole hyperplane $d_{2}(x)=0$.)

Now, let $D_{12}=m_{1}-m_{2}$. Then $D_{12}$ is zero on $d_{1}(x)=0$, because $D_{12}>0$ on $R_{1}$ and $D_{12}<0$ on $R_{2} \cup R_{3}$. As a consequence of Hilbert's Nullstellensatz $d_{1}$ is a factor of $D_{12}$. Therefore, there exists a polynomial function $e$ such that

$$
D_{12}=d_{1} e .
$$

Since $D_{12}$ is at most quadratic by assumption, we conclude that $e$ is a constant or a linear function. However, since both $d_{1} e$ and $d_{1}$ are positive on $R_{1}$ and negative on $R_{2}$, the function $e$ is positive on $R_{1} \cup R_{2}$. Since the degree of $e$ is $\leq 1, e$ must be a positive constant. 
Similarly, we have $D_{13}=d_{1} f$, where $f$ is a positive constant. Therefore $D_{23}=d_{1}(f-e)$. This contradicts the fact that $D_{23}$ is zero on $d_{2}(x)=0$.

Finally we discuss a small example, to show that multivariate polynomials can represent membership functions, although the degree of these polynomials is at least 5 .

Example Let $R_{1}, R_{2}$ and $R_{3}$ be the plane regions as given in figure 7 (right side). Then using the same notation as in the above theorem, we can prove that the polynomial $D_{23}$ has the form $D_{23}=x y g$, where $g$ is a polynomial of even degree. The reader can verify that the regions $R_{i}$ can be separated by the following polynomials of degree at most 5:

$$
\begin{aligned}
& m_{1}=0 \\
& m_{2}=x\left(y^{4}+1\right) \\
& m_{3}=x\left(y^{4}+y^{3}+1\right) .
\end{aligned}
$$

\section{An approximated mapping of a decision tree onto a one layer neural network}

Let $H_{k}$ be the hyperplane given by $d_{k}(x)=\sum_{j=1}^{n} w_{k j} x_{j}+w_{k 0}$, for $x=\left(x_{1}, \ldots, x_{n}\right) \in \mathbb{R}^{n}, k \in \mathcal{N}$. Then the distance of a point $x$ to $H_{k}$ is given by

$$
\left|w_{k}^{T} x+w_{k 0}\right| /|| w_{k}||, \text { where } w_{k}^{T}=\left(w_{k 1}, \ldots, w_{k n}\right) .
$$

Without restriction we may assume that $\left\|w_{k}\right\|=1$. Therefore, $\left|d_{k}(x)\right|$ is the distance of a point $x$ to the hyperplane $H_{k}$. We will show in this section that the difficulties encountered in the preceding section may be circumvented by requiring that the points we consider are not too close to the hyperplanes associated with the decision tree.

Let $(\mathcal{N}, \mathcal{L}, \mathcal{D})$ be a decision tree. We will restrict the regions $R_{\ell}$ by assuming that $\forall k \in \mathcal{N}$ : $0<e \leq\left|d_{k}(x)\right| \leq E$, where $e$ and $E$ are positive constants. The set of points in $R_{\ell}$ satisfying this condition will be denoted by $S_{\ell}$. Hence, $S_{\ell}$ is a convex subregion of $R_{\ell}$. Note also that $R_{\ell}$ can be approximated by $S_{\ell}$ with arbitrary precision, by varying the constants $e$ and $E$.

In [Par94] Park considers the following set of linear membership functions:

$$
m_{\ell}(x)=\sum_{k \in P_{\ell}} s_{\ell k} c_{k} d_{k}(x),
$$

where $P_{\ell}$ is the set of nodes on the path from the root to the leaf $\ell$. The constants $s_{\ell k}$ are defined as:

$$
s_{\ell k}= \begin{cases}+1 & \text { if } d_{k}(x)>0, \forall x \in R_{\ell} \\ -1 & \text { if } d_{k}(x)<0, \forall x \in R_{\ell}\end{cases}
$$

In this way it is secured that $\forall x \in R_{\ell}, \forall k \in P_{\ell}$ :

$$
s_{\ell k} d_{k}(x)>0 \text {. }
$$

The constants $c_{k}$ are determined experimentally in [Par94]. Here we will derive an explicit expression for these constants. Since as we have shown above that in general these constants cannot exist if $x \in R_{\ell}, \ell \in \mathcal{L}$, we will now assume that $x \in S_{\ell}$. 
Theorem 1 Let $(\mathcal{N}, \mathcal{L}, \mathcal{D})$ be a decision tree. Then there exists a set of linear functions $M=\left\{m_{\ell}, \ell \in \mathcal{L}\right\}$, such that for any $\ell, \ell^{\prime} \in \mathcal{L}$, with $\ell \neq \ell^{\prime}$ :

$$
\forall x \in S_{\ell}: m_{\ell}(x)>m_{\ell^{\prime}}(x) .
$$

Proof Let $\mathcal{T}$ be the set of terminal nodes of the tree. An internal node $t$ is called terminal if both children of $t$ are leaves. Further, if $n_{1}$ and $n_{2}$ are two nodes, then we write $n_{1}<n_{2}$ if $n_{1}$ is an ancestor of $n_{2}$. Suppose that $n_{a} \notin \mathcal{T}$, and $\ell, \ell^{\prime}$ are two leaves such that $n_{a}$ is the last common ancestor of $\ell$ and $\ell^{\prime}$, see figure 8 :

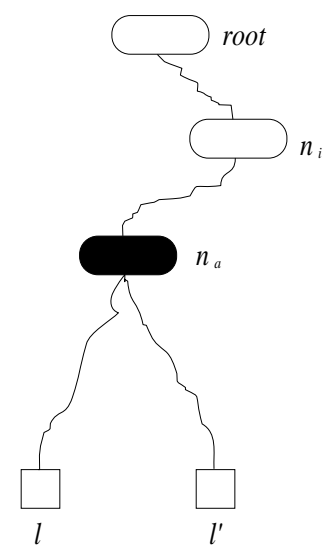

Figure 8: The last common ancestor of $\ell$ and $\ell^{\prime}$

We decompose the function $m_{\ell^{\prime}}$ as follows:

$$
m_{\ell^{\prime}}(x)=\sum_{n_{i}<n_{a}} s_{\ell i} c_{i} d_{i}(x)+\sum_{n_{j} \geq n_{a}} s_{\ell^{\prime} j} c_{i} d_{j}(x),
$$

where $n_{i} \in P_{\ell}$ and $n_{j} \in P_{\ell^{\prime}}$. The following crucial observation

$$
s_{\ell a}=-s_{\ell^{\prime} a}
$$

can be seen by applying (6) and (7) to the node $n_{a}$. From this we conclude:

$$
m_{\ell}(x)-m_{\ell^{\prime}}(x)=2 s_{\ell a} c_{a} d_{a}(x)+\Sigma_{1}-\Sigma_{2},
$$

where

$$
\Sigma_{1}=\sum_{n_{i}>n_{a}} s_{\ell i} c_{i} d_{i}(x), \text { with } n_{i} \in P_{\ell}
$$

and

$$
\Sigma_{2}=\sum_{n_{j}>n_{a}} s_{\ell^{\prime} j} c_{j} d_{j}(x), \text { with } n_{j} \in P_{\ell^{\prime}} .
$$

To assure that (8) holds, we require that

$$
\frac{\Sigma_{2}-\Sigma_{1}}{2 s_{\ell a} d_{a}(x)}<c_{a}
$$


Since $\Sigma_{1}$ is a positive number we can satisfy condition (9) by taking

$$
\frac{\Sigma_{2}}{2 s_{\ell a} d_{a}(x)}<c_{a}
$$

or

$$
\frac{E}{2 e} \sum_{n_{j}>n_{a}} c_{j} \leq c_{a} .
$$

This yields the following sufficient condition for the constants $c_{j}$ :

$$
\frac{E}{2 e} \max _{\ell \in \mathcal{L}_{a}}\left\{\sum_{n_{j}>n_{a}} c_{j}\right\} \leq c_{a},
$$

where $n_{j} \in P_{\ell}$ and $\mathcal{L}_{a}$ is the set of leaves of the subtree with root $n_{a}$. Condition (10) implies: $c_{a} \geq \frac{E}{2 e} \sum_{n_{j}>n_{a}} c_{j}$, where $n_{j} \in \mathcal{P}$ and $\mathcal{P}$ is the longest possible path from node $n_{a}$ to some leaf. From condition (10) it also follows that the constants $c_{a}$ are determined up to a constant factor. It is easy to see that the constants can be recursively determined by choosing positive values $c_{t}$ for the set of terminal nodes $t \in \mathcal{T}$. As an example we show in figure 9 the values of all the constants $c_{j}$ for a tree, when we take $c_{t}=1$ for all $t \in \mathcal{T}$ and $\frac{E}{2 e}=1$.

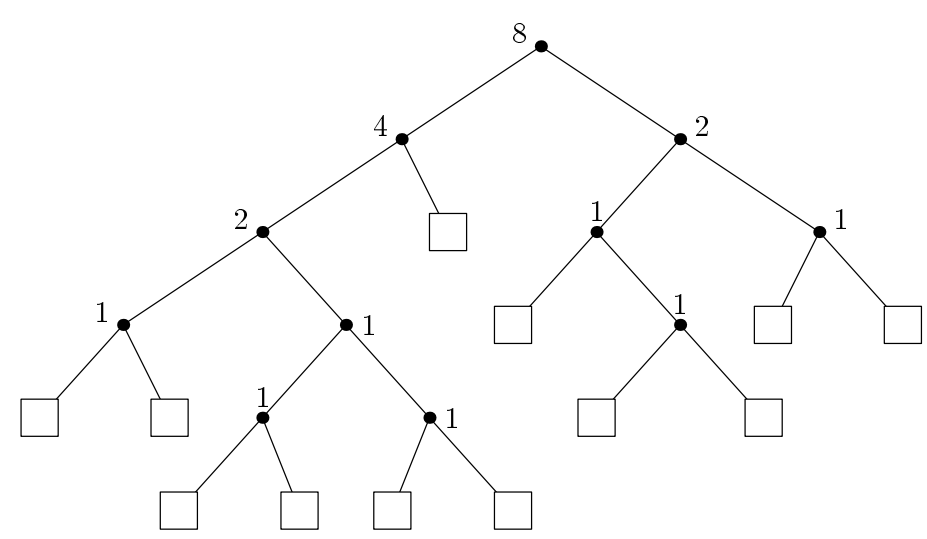

Figure 9: Determination of constants $c_{j}$ in an example tree.

To derive an an explicit expression for $c_{a}$ for any node $n_{a}$ we will introduce some notation. Let $\mathcal{P}_{a}=\left\{t=n_{0}<n_{1}<n_{2}<\ldots<n_{k}=n_{a}\right\}$ be the longest possible path from node $n_{a}$ to a terminal node $t$. The length $k$ of $\mathcal{P}_{a}$ is denoted by $\delta(a)$. Similarly, $\delta(r)$ is the length of the longest path starting in the root $r$ of the tree.

Lemma Let $\left(a_{i}\right)_{i=0}^{\infty}$ be the sequence defined by $a_{k}=\lambda\left(a_{0}+a_{1}+\ldots+a_{k-1}\right)$ for $k \geq 2$ and $a_{1}=\lambda a_{0}$. Then $a_{k}=a_{0} \lambda(\lambda+1)^{k-1}, k \geq 1$.

Proof For $k \geq 2$ we have: $a_{k+1}-a_{k}=\lambda a_{k}$. This implies $a_{k}=(1+\lambda)^{k-1} a_{1}=\lambda a_{0}(1+$ $\lambda)^{k-1}, k \geq 1$.

Theorem 2 The constants

$$
c_{a}=\left(\frac{1}{1+\frac{E}{2 e}}\right)^{\delta(r)-\delta(a)}
$$


satisfy condition (10).

Proof Let $A_{a}=\frac{E}{2 e} \max _{\ell \in \mathcal{L}_{a}}\left\{\sum_{j \in P_{\ell}} A_{j}\right\}$, and $A_{\ell}=1$. Then $A_{a}=\lambda(1+\lambda)^{\delta(a)-1}$ (see the above Lemma), with $\lambda=\frac{E}{2 e}$. Since the constants $c_{a}$ are defined up to a constant factor, we choose $c_{a}=\frac{A_{a}}{A_{r}}$. Then $c_{a}=\left(\frac{1}{1+\lambda}\right)^{\delta(r)-\delta(a)}$.

Corollary Let $\rho=1 /\left(1+\frac{E}{2 e}\right)$. Then $m_{\ell}(x)=\sum_{k} s_{k \ell} \rho^{\delta(r)-\delta(k)} d_{k}(x)$ are linear functions for which (8) holds.

Another expression for the constants $c_{a}$ can be obtained by using the fact that a decision tree $(\mathcal{N}, \mathcal{L}, \mathcal{D})$ in practical situations is derived from a finite set of examples $V \subset \mathbb{R}^{n}$. It is no restriction to assume that $\forall x \in V:\left|d_{k}(x)\right|>0, k \in \mathcal{N}$.

Let $M=\max _{i, j} \max _{x \in V}\left|\frac{d_{i}(x)}{d_{j}(x)}\right|$, where $n_{i}, n_{j} \in \mathcal{N}$ and $n_{i}<n_{j}$. The number $M$ can be easily computed from the set of examples $V$.

Theorem 3 Let $\rho=2 /(3 M)$. Then the constants $c_{a}=\rho^{\delta(r)-\delta(a)}$ satisfy condition (10).

Proof Suppose $P_{\ell}$ is the longest path from node $n_{a}$ to a leaf $\ell$, and $n_{j}, n_{j+1}$ are two consecutive nodes on $P_{\ell}$ with $n_{j}<n_{j+1}$. Then $d_{j}(x) \leq M d_{j+1}(x)$, so that $d_{j}(x) \leq M^{\delta(a)-\delta(j)}\left|d_{a}(x)\right|$. To satisfy condition (9) we want to choose constants $A_{j}$ such that

$$
\Sigma=\sum_{n_{j}>n_{a}} \frac{s_{m j} A_{j} d_{j}(x)}{2 s_{\ell a} d_{a}(x)} \leq A_{a} .
$$

Since

$$
\Sigma \leq \frac{1}{2} \sum_{j} \frac{A_{j}}{M^{\delta(j)}} M^{\delta(a)} \leq A_{a}
$$

we have

$$
\frac{1}{2} \sum_{j} \frac{A_{j}}{M^{\delta(j)}} \leq \frac{A_{a}}{M^{\delta(a)}},
$$

or $\frac{1}{2} \sum_{j} B_{j} \leq B_{a}$, where $B_{j}=A_{j} / M^{\delta(j)}$. Now we choose $B_{a}$ such that $B_{a}=\frac{1}{2} \sum_{j} B_{j}$. Then by the Lemma preceding Theorem 2 we have $B_{a}=\lambda(\lambda+1)^{\delta(a)-1}$, with $\lambda=\frac{1}{2}$. Finally, let $c_{a}=B_{a} / B_{r}$; then we have

$$
c_{a}=\left(\frac{1}{M(\lambda+1)}\right)^{\delta(r)-\delta(a)}=\rho^{\delta(r)-\delta(a)}, \text { with } \rho=\frac{2}{3 M} .
$$

Discussion: We have found two possible values for $\rho_{\text {: }} \rho_{1}=1 /(1+E /(2 e))$ and $\rho_{2}=2 /(3 M)$. From the definition of $M$ it follows that $M \leq E / e$. Since $E / e \geq 1$, we have $\rho_{1} \leq 1 /\left(1+\frac{1}{2}\right)=$ $2 / 3$. On the other hand, it is easy to verify that $\rho_{1} \geq \rho_{2}$ if and only if $E / e \leq 3 M-2$.

Since $\rho \rightarrow 0$ if $e \rightarrow 0$, in practice we will choose the parameter $e$ not too small. On the other hand, the approximation of the regions $R_{\ell}$ by $S_{\ell}$ will be less accurate if $e$ becomes larger. In a tree to neural net mapping the functions $m_{\ell}$ are used to define the initial weights between the input and hidden layer. To improve the performance of the neural net these weights are subsequently updated. It is therefore plausible, and in accordance with some experiments of Park [Par94], and with the data used in an experiment described in [Car95] that the accuracy of the approximation of the regions $R_{\ell}$ must be less, the more the data set used to generate the decision tree is non-linear. 


\section{Another initialisation method}

In this section we consider another well-known classifier: Learning Vector Quantisation (LVQ) networks. This method can be used to solve a classification problem with $m$ classes and data-vectors $x \in \mathbb{R}^{n}$. It is known that the LVQ-network induces a so-called Voronoi tesselation of the input space, see [HKP91] chapter 9. Training of an LVQ-network yields prototype vectors $w_{j} \in \mathbb{R}^{n}, j=1, \ldots, m$ such that an input vector $x$ belongs to class $j$ iff the distance to $w_{j}$ is smallest:

$$
\forall i \neq j:\left\|w_{j}-x\right\| \leq\left\|w_{i}-x\right\| \Rightarrow x \in R_{j}
$$

It is easy to see that this is equivalent with

$$
w_{j}^{T} x-\frac{1}{2} w_{j}^{T} w_{j} \geq w_{i}^{T} x-\frac{1}{2} w_{i}^{T} w_{i}
$$

Now define the linear membership function $m_{i}$ as:

$$
m_{i}(x)=w_{i}^{T} x-\frac{1}{2} w_{i}^{T} w_{i}
$$

Then

$$
x \in R_{i} \Longleftrightarrow m_{i}(x)>m_{j}(x), \forall j \neq i .
$$

Since an LVQ-network is a relatively good classifier and can be trained relatively fast, it is a good alternative for the initialisation of a neural net using linear membership functions. Apparently, an LVQ-network cannot induce the convex regions induced by an LTC.

Example Let $R_{1}, R_{2}$ and $R_{3}$ be such as in figure 10 (right side). Now let $w_{1}$ be the prototype vector of $R_{1}$. Then the situation of figure 10 (left) can only be obtained if $\left\|w_{1}\right\| \rightarrow \infty$.
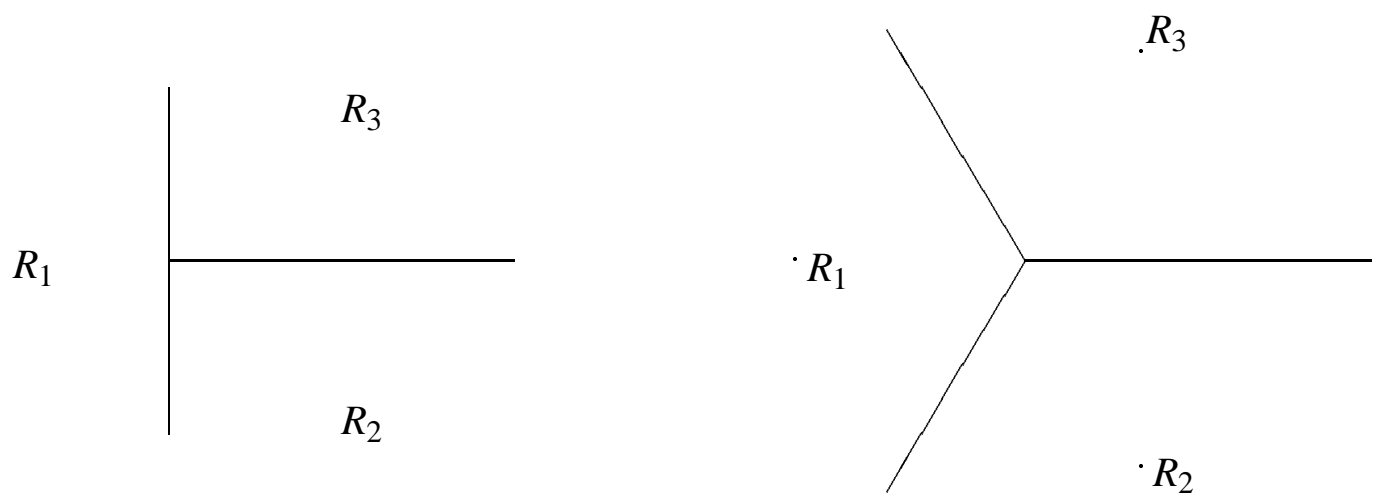

Figure 10: Different types of convex regions 


\section{Conclusions and further research}

We have proven that linear (or quadratic) membership functions representing the convex regions of a linear classifier in general do not exist. However, we give explicit formulae for the approximation of such functions. This allows us to control the degree of approximation. This is useful, for in practical applications the membership functions are 'only' used to initialise a one-hidden-layer neural net. Subsequently this net is further trained with a standard training algorithm. Currently we are investigating how to determine an appropriate approximation in a given application. We are also investigating two other approaches, see [Car95] to obtain a mapping from am LTC to a one-hidden-layer neural net. In the first approach we combine the first two layers of Sethi's net into one layer. The other approach uses appropriate transfer functions for the hidden layer to represent a join of two half spaces, because it is clear that an exact representation of the convex regions can be obtained by using piecewise linear functions. Finally, we have discussed LVQ-networks as an alternative that is of interest for the initialisation of the input-to-hidden layer based on membership functions of convex regions. This architecture divides the input space in polyhedral regions, see [MP88, HKP91] with linear membership functions.

\section{Acknowledgement}

We are grateful to Reino de Boer for his help with the pictures and advice on $\mathrm{LT}_{\mathrm{E}} \mathrm{X}$.

\section{References}

[BDV94] J.C. Bioch, M. van Dijk and W. Verbeke, Neural Networks: New Tools for Data Analysis?, In: M. Taylor and P. Lisboa (eds.) Proceedings of Neural Networks Applications and Tools, IEEE Computer Society Press, pp. 28-38, 1994.

[Car95] R. Carsouw, Learning to Classify: Classification by neural nets based on decision trees, Masterthesis (in Dutch), Dept. of Computer Science, Erasmus University Rotterdam, Februari 1995.

[IKP94] I. Ivanova, M. Kubat and G. Pfurtscheller, The System TBNN for Learning of 'Difficult' Concepts. In: J.C. Bioch and S.H. Nienhuys-Cheng (eds), Proceedings of Benelearn94, Tech. Rep. eur-09-94, Dept. of Computer Science, Erasmus University Rotterdam, pp. 230-241, 1994.

[FaI92] U.M. Fayad and K.B. Irani, On the Handling of Continuous-Valued Attributes in Decision Tree Generation, Machine Learning, vol. 8, pp. 88-102, 1992.

[HKP91] J. Hertz, A. Krogh, R.G. Palmer, Introduction to the theory of neural computation, Addison-Wesley, 1991.

[MP88] M.L. Minsky, S.A. Papert, Perceptrons, 2nd ed., MIT Press, 1988.

[Par94] Y. Park, A Mapping From Linear Tree Classifiers to Neural Net Classifiers, Proceedings of IEEE ICNN, vol. I, pp. 94-100, Orlando, Florida, 1994. 
[PaS90] Y. Park and J. Sklansky, Automated Design of Linear Tree Classifiers, Pattern Recognition, vol. 23, no. 12, pp. 1393-1412, 1990.

[Set90] A.K. Sethi, Entropy Nets: From Decision Trees to Neural Networks, Proceedings of the IEEE, vol. 78, no. 10, pp. 1606-1613, 1990.

[Qui93] J.R. Quinlan, C4.5 Programs for Machine Learning, Morgan Kaufmann, San Mateo, California, San Mateo, 1993. 